\title{
Eisenstein series on four-dimensional hyperbolic space
}

\author{
by \\ Valeri A. Gritsenko (St. Petersburg) \\ and Rainer Schulze-Pillot (Köln)
}

In this paper we continue the investigation of Eisenstein series on the four-dimensional hyperbolic space that was started in [Gr1]. Our main goal is to give an explicit computation of the Fourier coefficients of the standard Eisenstein series and to use this for the study of some new and interesting Dirichlet series arising as Mellin transforms of the Eisenstein series and of its pullbacks to embedded hyperbolic spaces of smaller dimension. Studying these pullbacks seems also to be of some interest for giving explicit constructions of automorphic forms on these domains and we give some examples of square integrable forms obtained in this way (which are, however, not eigenfunctions of the Laplacian).

In Section 1 we review basic facts and notation. In particular, we realize (as usual) the four-dimensional hyperbolic space as the set of Hamilton quaternions of positive trace on which the unitary group of the standard split two-dimensional hermitian form over the Hamilton quaternions acts. We consider here arithmetic subgroups given as subgroups of matrices with entries in a maximal order $R$ of some (fixed) definite quaternion algebra over the rationals. The inequivalent cusps are then in bijection with the classes of left ideals of $R$ and we consider the Eisenstein series formed with respect to each cusp. The computation of the Fourier coefficients of these Eisenstein series is given in Section 2. It turns out that a weighted average of these Eisenstein series has Fourier coefficients of a particularly simple and interesting form: The value of the arithmetic part of the Fourier coefficient at $s$ is expressed in terms of values at $s-1$ of Cohen-Zagier $L$-functions. This should be compared with the case of Eisenstein series on 3-dimensional hyperbolic space treated in [EGM], where there is only a divisor sum expressions. As a corollary we obtain the meromorphic continuation of this Eisenstein series, which of course also follows from the work of Langlands.

Partially supported by SFB 343 University of Bielefeld and SFB 170 University of Göttingen. 
We get the exact form of the functional equation and an explicit calculation of the poles and residues. As another corollary we receive asymptotics for sums of the Fourier coefficients which give rise to asymptotic formulae for sums of class numbers.

In Section 3 we consider the Mellin transform of the Eisenstein series which leads to a Dirichlet series whose coefficients are products of representation numbers of ternary quadratic forms and of sums of special values of Cohen-Zagier $L$-functions. By considering suitable linear combinations of the Eisenstein series associated with different conjugacy classes of maximal orders in the same quaternion algebra over $\mathbb{Q}$ we obtain similarly Dirichlet series whose coefficients are products of Fourier coefficients of modular forms of half integral weight and of sums of special values of Cohen-Zagier $L$ functions. Since these Dirichlet series come from the Eisenstein series we can give functional equations and meromorphic continuations for them. From this we get again asymptotic formulae for products of the type described above.

In the final Section 4 we restrict our Eisenstein series to embedded 2and 3-dimensional hyperbolic spaces. Comparing (in the 2-dimensional case) the restrictions of Eisenstein series with respect to different maximal orders we obtain square integrable automorphic forms with respect to congruence subgroups of type $\Gamma_{0}(p)$ with explicitly given Fourier expansions (which are, however, not eigenfunctions of the Laplacian). Although we have not yet been able to obtain cusp forms by this method, it seems possible that these might be constructed using the techniques which we develop here. The Mellin transforms of the Eisenstein series lead to Dirichlet series with meromorphic continuations and functional equations whose coefficients are products of representation numbers of binary quadratic forms and sums of values of Cohen-Zagier $L$-functions.

1. Basic facts and notation. Let $B$ be a definite quaternion algebra over $\mathbb{Q}$; let $\mathbb{H}=B \otimes \mathbb{R}$ be the Hamilton quaternions. By $x \mapsto \bar{x}$ we denote the standard involution; by $\operatorname{tr}$ and $n$ the reduced trace and norm in $B$; by $z_{r}$ the "real part" $\frac{1}{2} \operatorname{tr} z$ of $z \in B$, we put $B^{(0)}=\{x \in B \mid \operatorname{tr} x=0\}$. Let $G$ be the unitary group of the 2-dimensional hermitian form with matrix $\left(\begin{array}{ll}0 & 1 \\ 1 & 0\end{array}\right)$ over $B$, that is,

$$
G=\left\{\left(\begin{array}{ll}
a & b \\
c & d
\end{array}\right) \in M_{2}(B) \mid\left(\begin{array}{ll}
a & b \\
c & d
\end{array}\right) \text { is inverse to }\left(\begin{array}{cc}
\bar{d} & \bar{b} \\
\bar{c} & \bar{a}
\end{array}\right)\right\} .
$$

The group $G_{\mathbb{R}}$ is also called the modified symplectic group $\operatorname{MSp}(1, \mathbb{H})$; it operates on the 4-dimensional hyperbolic space $\mathcal{H}=\{z \in \mathbb{H} \mid \operatorname{tr} z>0\}$ by fractional linear transformations, i.e., $g=\left(\begin{array}{ll}a & b \\ c & d\end{array}\right)$ operates by $z \mapsto g z=$ $(a z+b)(c z+d)^{-1}$. If $R$ is a maximal order in $B$ we let $\Gamma_{R} \subset G_{\mathbb{Q}}$ be 
the set of $g \in G_{\mathbb{Q}}$ such that $g$ and $g^{-1}$ have entries in $R$. This is known to be a discrete subgroup of $G_{\mathbb{R}}$ of finite covolume. From [Bo] (Prop. 7.5 and Prop. 2.7) it is clear that $\mathcal{H}$ has $h$ inequivalent cusps with respect to $\Gamma_{R}$, where $h$ is the number of classes of left $R$-ideals (this number does not depend on the choice of $R$ ). The correspondence between equivalence classes of cusps and ideal classes is given concretely by associating with the cusp $\mathfrak{c}=[a, b] \in \mathbb{P}^{1}(B)=B^{2} / B^{\times}$(multiplication by $B^{\times}$from the right) with $\operatorname{tr}(a \bar{b})=0$ the left $R$-ideal $I_{\mathfrak{c}}=R a+R b$, whose right order is denoted by $R_{\mathfrak{c}}$. Conversely, any left ideal $I$ of $R$ can be written as $I=R a+R b$ with $a, b \in I$ (see $[\mathrm{Ch}]$ ), and it is not difficult to see that $a, b$ can be chosen such that $\operatorname{tr}(a \bar{b})=0$ and that the equivalence class of the cusp $[a, b]$ is then uniquely determined by the class of the left ideal $I$.

For the cusp $\mathfrak{c}=[a, b]$ let $\left(\Gamma_{R}\right)_{\mathfrak{c}}$ denote the stabilizer of $\mathfrak{c}$ in $\Gamma_{R}$, let $\gamma_{\mathfrak{c}} \in G$ be such that $\gamma_{\mathfrak{c}}\left(\begin{array}{l}1 \\ 0\end{array}\right)=\left(\begin{array}{l}a \\ b\end{array}\right)$ and consider for $s \in \mathbb{C}$ and $z \in \mathcal{H}$ the Eisenstein series

$$
E_{\mathfrak{c}}(z, s):=\sum_{\gamma \in\left(\Gamma_{R}\right)_{\mathfrak{c}} \backslash \Gamma_{R}}\left(\frac{1}{2} \operatorname{tr}\left(\gamma_{\mathfrak{c}}^{-1} \gamma z\right)\right)^{s} .
$$

If $\gamma^{-1}\left(\begin{array}{l}a \\ b\end{array}\right)=\left(\begin{array}{l}a_{\gamma} \\ b_{\gamma}\end{array}\right)$ then $\gamma_{\mathfrak{c}}^{-1} \gamma=\left(\begin{array}{cc}{ }^{*} & { }^{*} \\ \bar{a}_{\gamma}\end{array}\right)$ and hence

$$
\left(\gamma_{\mathfrak{c}}^{-1} \gamma z\right)_{r}=\frac{z_{r}}{n\left(\bar{b}_{\gamma} z+\bar{a}_{\gamma}\right)}
$$

Since

$$
\Gamma_{R}\left(\begin{array}{l}
a \\
b
\end{array}\right)=\left\{\left(\begin{array}{l}
a^{\prime} \\
b^{\prime}
\end{array}\right) \in B^{2} \mid R a^{\prime}+R b^{\prime}=R a+R b, \operatorname{tr}\left(a^{\prime} \overline{b^{\prime}}\right)=0\right\}
$$

and since

$$
\gamma \mapsto \gamma^{-1}\left(\begin{array}{l}
a \\
b
\end{array}\right)=\left(\begin{array}{l}
a_{\gamma} \\
b_{\gamma}
\end{array}\right)
$$

gives a bijection from $\left(\Gamma_{R}\right)_{\mathfrak{c}} \backslash \Gamma_{R}$ onto $\Gamma_{R}\left(\begin{array}{l}a \\ b\end{array}\right) / R_{\mathfrak{c}}^{\times}$, we see that

$$
E_{\mathfrak{c}}(z, s)=z_{r}^{s} \sum(n(\bar{a} z+\bar{b}))^{-s},
$$

where the sum is over all pairs $(a, b) \in\left(I_{\mathfrak{c}} \times I_{\mathfrak{c}}\right) / R_{\mathfrak{c}}^{\times}$such that $R a+R b=I_{\mathfrak{c}}$ and $\operatorname{tr}(a \bar{b})=0$.

As usual in the theory of Eisenstein series it is easier to deal with the related series

$$
\widetilde{E}_{\mathfrak{c}}(z, s)=z_{r}^{s} \sum^{\prime} n(\bar{a} z+\bar{b})^{-s}
$$

in which the sum runs over all pairs $(a, b) \in I_{\mathfrak{c}} \times I_{\mathfrak{c}}$ with $\operatorname{tr}(a \bar{b})=0$. In order to give the relation between the two series we introduce some more notation; for the underlying facts from the theory of quaternion algebras see [Vi].

Let $I_{1}=R, I_{2}, \ldots, I_{h}$ be a set of representatives of the classes of left $R$-ideals in $B$, and denote by $R_{i}$ the right order $R_{i}=\left\{a \in B \mid I_{i} a \subset I_{i}\right\}$. It 
is known that one can choose the $I_{i}$ such that $n\left(I_{i}\right) \mathbb{Z}=\mathbb{Z}$; we will always do so in the sequel. Let $I_{i j}(1 \leq i, j \leq h)$ be given by $I_{i j}=I_{i}^{-1} I_{j}$, where for a left $R$-ideal $I$ the inverse $I^{-1}$ is the right $R$-ideal $I^{-1}=\{a \in B \mid I a \subset R\}$ whose left order is the right order of $I$. The ideal $I_{i j}$ has then left order $R_{i}$ and right order $R_{j}$, and one has $I_{1 j}=I_{j}$ for all $j$. Let

$$
Z\left(I_{i j}, s\right)=\sum_{0 \neq x \in I_{i j}} n(x)^{-s}=e_{i} \sum_{m=1}^{\infty} B_{j i}(m) m^{-s}
$$

with $B_{i j}(m)$ denoting the entries of the Brandt matrix and $e_{i}=\left|R_{i}^{\times}\right|$; the Brandt matrix series $\sum_{m=1}^{\infty} B_{i j}(m) m^{-s}$ is also written as $B_{i j}(s)$. Let $\mathfrak{c}_{1}, \ldots, \mathfrak{c}_{h}$ be representatives of the $\Gamma_{R}$-equivalence classes of cusps of $\mathcal{H}$, corresponding to $I_{1}, \ldots, I_{h}$ as described above; write $\gamma_{\mathfrak{c}_{i}}=\gamma_{i}$ and $E_{i}(z, s):=$ $E_{\mathfrak{c}_{i}}(z, s), \widetilde{E}_{i}(z, s):=\widetilde{E}_{\mathfrak{c}_{i}}(z, s)$.

If we perform the same constructions starting with the right order $R_{j}$ of the ideal $I_{j}$ instead of $R$, then the $I_{j i}=\bar{I}_{j} I_{i}(i=1, \ldots, h)$ are a set of representatives of the classes of left $R_{j}$-ideals, and to the ideal $I_{j i}$ there corresponds the cusp $\mathfrak{c}_{j i}:=\gamma_{j}^{-1} \gamma_{i}\left(\begin{array}{l}1 \\ 0\end{array}\right)$ of $\Gamma_{R_{j}}$. We then get the Eisenstein series $E_{j i}^{(j)}(z, s)$ and $\widetilde{E}_{j i}^{(j)}(z, s)$ with $E_{1 i}^{(1)}(z, s)=E_{i}(z, s), \widetilde{E}_{1 i}^{(1)}(z, s)=\widetilde{E}_{i}(z, s)$ and notice:

LEMMA 1.1. With notations as above one has

$$
E_{j i}^{(j)}(z, s)=E_{i}\left(\gamma_{j} z, s\right), \quad \widetilde{E}_{j i}^{(j)}(z, s)=\widetilde{E}_{i}\left(\gamma_{j} z, s\right) .
$$

Proof. We have

$$
\begin{aligned}
E_{i}\left(\gamma_{j} z, s\right) & =\sum_{\gamma \in\left(\Gamma_{R}\right)_{\mathfrak{c}_{i} \backslash \Gamma_{R}}}\left(\gamma_{i}^{-1} \gamma \gamma_{j} z\right)_{r}^{s} \\
& =\sum_{\gamma \in\left(\Gamma_{R}\right)_{\mathfrak{c}_{i} \backslash \Gamma_{R}}}\left(\gamma_{i}^{-1} \gamma_{j} \gamma_{j}^{-1} \gamma \gamma_{j} z\right)_{r}^{s}=\sum_{\gamma}\left(\gamma_{i}^{-1} \gamma_{j} \gamma z\right)_{r}^{s},
\end{aligned}
$$

where the sum now is over $\gamma \in \gamma_{j}^{-1}\left(\Gamma_{R}\right)_{\mathfrak{c}_{i}} \gamma_{j} \backslash \gamma_{j}^{-1} \Gamma_{R} \gamma_{j}$. Since $\gamma_{j}^{-1} \Gamma_{R} \gamma_{j}=\Gamma_{R_{j}}$ and

$$
\gamma_{j}^{-1}\left(\Gamma_{R}\right)_{\mathfrak{c}_{i}} \gamma_{j}=\gamma_{j}^{-1} \Gamma_{R} \gamma_{j} \cap \gamma_{j}^{-1} \gamma_{i} \Gamma_{\infty} \gamma_{i}^{-1} \gamma_{j}=\left(\Gamma_{R_{j}}\right)_{\mathfrak{c}_{j i}},
$$

the assertion for $E_{i}(z, s)$ is obvious. The assertion for $\widetilde{E}_{i}$ can be deduced similarly or as a consequence of the following lemma:

LEMma 1.2. The series $\widetilde{E}_{i}(z, s)$ and $E_{i}(z, s)$ are absolutely convergent for $\operatorname{Re} s>3$. With the notations introduced above one has, in this domain,

$$
\widetilde{E}_{j}(z, s)=\sum_{i=1}^{h} Z\left(I_{i j}, s\right) E_{i}(z, s) .
$$


Proof. The convergence assertion is obvious. The rest of the proof proceeds as in [EGM]: If $(a, b) \in S_{i}=\left\{(a, b) \in I_{i} \times I_{i} \mid R a+R b=I_{i}, \operatorname{tr}(a \bar{b})=0\right\}$ and $c \in B^{\times} \cap I_{i j}$ then $\left(a^{\prime}, b^{\prime}\right)=(a c, b c) \in I_{j} \times I_{j}$.

The mapping $\bigcup_{i=1}^{h}\left(S_{i} \times\left(B^{\times} \cap I_{i j}\right)\right) \rightarrow I_{j} \times I_{j} \backslash\{(0,0)\}$ given thus by $((a, b) ; c) \mapsto(a c, b c)$ is surjective since for $\left(a^{\prime}, b^{\prime}\right) \in I_{j} \times I_{j}$ one has $R a^{\prime}+R b^{\prime}=$ $I_{i} c$ for some $i \in\{1, \ldots, h\}$ and some $c \in B^{\times} \cap I_{i j}$, hence $\left(a^{\prime}, b^{\prime}\right)$ is the image of $\left(\left(a^{\prime} c^{-1}, b^{\prime} c^{-1}\right) ; c\right)$. Since each element of $I_{j} \times I_{j}$ has $e_{i}=\left|R_{i}^{\times}\right|$preimages under this mapping, we have for each summand $n(\bar{a} z+\bar{b})^{-s}$ in $E_{i}$ and for each $m \in \mathbb{N}$ precisely $\#\left\{c \in I_{i j} \mid n(c)=m\right\}$ terms $m^{-s} n(\bar{a} z+\bar{b})^{-s}$ in $\widetilde{E}_{j}$, which is just our assertion.

From the works of Eichler [E] it is known that the Brandt matrix series can be diagonalized so that the diagonal entries are

$$
\zeta(s) \zeta(s-1) \prod_{p \mid D}\left(1-p^{1-s}\right)
$$

and the Hecke $L$-functions $L\left(f_{i}, s\right)$ of the normalized cuspidal eigenforms of weight 2 and level $D$ of all Hecke operators (here $D$ denotes the discriminant of the quaternion algebra $B$ ). Therefore:

LEMMA 1.3. The series $E_{i}(z, s)$ and $\widetilde{E}_{i}(z, s)$ span the same space of functions on $\mathcal{H}$ for $\operatorname{Re} s>3$ and (after analytic continuation) even for $\operatorname{Re} s>1$ with the exception of $s=2$, where the residues of the $\widetilde{E}_{i}$ span the same space as the $E_{i}$.

2. Computation of Fourier coefficients. We now proceed to compute the Fourier coefficients of the series $\widetilde{E}_{i}(z, s)$ introduced in Section 1. By Lemma 1.1 we may restrict our attention to expansions about infinity.

Lemma 2.1. For $\operatorname{Re} s>3$ the series $\widetilde{E}_{i}(z, s)$ has the Fourier expansion

$$
\widetilde{E}_{i}(z, s)=z_{r}^{s} Z\left(I_{i}, s\right)+\frac{2}{D} \sum_{u \in R^{(0)}} a_{u}\left(z_{r}, s\right) b_{u}^{(i)}(s) \exp (2 \pi i \operatorname{tr}(\bar{u} z)) .
$$

Here $R^{(0)}=R \cap B^{(0)}$ is the sublattice of the order $R$ consisting of the quaternions with trace zero and $\widehat{R^{(0)}}=\left\{x \in B^{(0)} \mid \operatorname{tr}\left(x R^{(0)}\right) \subset \mathbb{Z}\right\}$ is its dual lattice,

$$
a_{u}\left(z_{r}, s\right)= \begin{cases}\pi^{3 / 2} z_{r}^{3-s} \Gamma(s-3 / 2) \Gamma(s)^{-1} & \text { if } u=0, \\ 2 \pi^{s} \Gamma(s)^{-1} n(2 u)^{(2 s-3) / 4} z_{r}^{3 / 2} K_{s-3 / 2}\left(2 \pi z_{r} \sqrt{n(2 u)}\right) & \text { if } u \neq 0,\end{cases}
$$

and

$$
b_{u}^{(i)}(s)=\sum n(c)^{-s} \exp \left(2 \pi i \operatorname{tr}\left(\bar{u} c^{-1} d\right)\right),
$$


where the summation is over $c \in \bar{I}_{i}, c \neq 0$ and $d \in\left(\bar{I}_{i} \cap c B^{(0)}\right) / c R^{(0)}$ and $K_{s}$ denotes the modified Bessel function

$$
K_{s}(x)=\frac{1}{2} \int_{0}^{\infty} \exp \left(-\frac{x}{2}\left(t+t^{-1}\right)\right) t^{s-1} d t
$$

Proof. Since $\widetilde{E}_{i}$ is invariant under translations $z \mapsto z+z^{\prime}$ with $z^{\prime} \in R^{(0)}$ it has a Fourier expansion

$$
\widetilde{E}_{i}(z, s)=\sum_{u \in \widehat{R}^{(0)}} f_{u}^{(i)}\left(z_{r}, s\right) \exp (2 \pi i \operatorname{tr}(\bar{u} z))
$$

with

$$
f_{u}^{(i)}\left(z_{r}, s\right)=\frac{1}{\mu\left(B^{(0)} / R^{(0)}\right)} \int_{B^{(0)} / R^{(0)}} \widetilde{E}_{i}(z, s) \exp (-2 \pi i \operatorname{tr}(\bar{u} z)) d \mu
$$

where $B^{(0)} / R^{(0)}$ is a fundamental parallelepiped for the lattice $R^{(0)}$ in the real vector space $B_{\mathbb{R}}^{(0)}$ and $d \mu$ is the Lebesgue measure on $B_{\mathbb{R}}^{(0)}$. In the domain $\{s \mid \operatorname{Re} s>3\}$ of absolute convergence of the series we compute $f_{u}^{(i)}\left(z_{r}, s\right)$ as usual by interchanging summation and integration and get

$$
\begin{aligned}
& f_{u}^{(i)}\left(z_{r}, s\right) \\
& =z_{r}^{s} \sum_{b \in I_{i}} n(b)^{-s}+\frac{1}{\mu\left(B^{(0)} / R^{(0)}\right)} \sum_{0 \neq a \in I_{i}} n(a)^{-s} \\
& \quad \times \sum_{\bar{b} \in \bar{I}_{i} \cap\left(\bar{a} B^{(0)} / \bar{a} R^{(0)}\right)} \sum_{\ell \in R^{(0)}} \int_{B^{(0)} / R^{(0)}} \frac{z_{r}^{s}}{n\left(z+\bar{a}^{-1} \bar{b}+\ell\right)^{s}} \exp (-2 \pi i \operatorname{tr}(\bar{u} z)) d \mu \\
& =z_{r}^{s} Z\left(I_{i}, s\right)+\frac{1}{\mu\left(B^{(0)} / R^{(0)}\right)} \sum_{0 \neq c \in \bar{I}_{i}} n(c)^{-s} \\
& \quad \times \sum_{d \in\left(c B^{(0)} \cap \bar{I}_{i}\right) / c R^{(0)}} \exp \left(2 \pi i \operatorname{tr}\left(\bar{u} c^{-1} d\right)\right) \int_{B^{(0)}} \frac{z_{r}^{s}}{n(z)^{s}} \exp (-2 \pi i \operatorname{tr}(\bar{u} z)) d \mu,
\end{aligned}
$$

where the first summands appear only if $u=0$. Denoting by $z_{r}, z_{1}, z_{2}, z_{3}$ the coordinates of $z$ with respect to the usual basis of the Hamilton quaternions the last integral is

$$
\begin{aligned}
a_{u}(z, s) & \\
\quad & :=\int_{\mathbb{R}^{3}} \frac{z_{r}^{s}}{\left(z_{r}^{2}+z_{1}^{2}+z_{2}^{2}+z_{3}^{2}\right)^{s}} \exp \left(-4 \pi i\left(u_{1} z_{1}+u_{2} z_{2}+u_{3} z_{3}\right)\right) d z_{1} d z_{2} d z_{3}
\end{aligned}
$$

which we evaluate by rotating $u=\left(u_{1}, u_{2}, u_{3}\right)$ onto the point $\left(n(u)^{1 / 2}, 0,0\right)$. 
We then apply the well known relation

$$
\int_{-\infty}^{\infty} \frac{\exp (-2 \pi i y x)}{\left(t^{2}+x^{2}\right)^{s}} d x= \begin{cases}2 \pi^{s}\left|y t^{-1}\right|^{s-1 / 2} \Gamma(s)^{-1} K_{s-1 / 2}(2 \pi|y t|) & \text { if } y \neq 0 \\ \pi^{1 / 2}|t|^{1-2 s} \Gamma(s)^{-1} \Gamma(s-1 / 2) & \text { if } y=0\end{cases}
$$

$K_{s}$ denoting the modified Bessel function as above.

This gives the asserted value of $a_{u}(z, s)=a_{u}\left(z_{r}, s\right)$. We finally notice that the lattice $R^{(0)}$ in $B_{\mathbb{R}}^{(0)}=\mathbb{R}^{3}$ has discriminant $D^{2} / 4$ with respect to the standard bilinear form $B(x, y)=\sum_{i=1}^{3} x_{i} y_{i}$ on $\mathbb{R}^{3}$, hence $\mu\left(B^{(0)} / R^{(0)}\right)=$ $D / 2$.

The "arithmetical part" $b_{u}^{(i)}(s)$ of the Fourier coefficients of the $\widetilde{E}_{i}(z, s)$ was calculated in [Gr1] in the case of the quaternion algebra with discriminant $D=2$. Professor D. Zagier found an elegant form of the main formula for $b_{u}(s)$ in [Gr1] using the special $L$-functions defined in [Za2]. We follow here his idea.

We need the following two elementary lemmas:

Lemma 2.2. For $c \in B^{\times}, d \in B$ and $m \in n(c) \mathbb{Z}$ one has

$$
\sum_{\mu \in \mathbb{Z} / m \mathbb{Z}} \exp \left(2 \pi i \operatorname{tr}\left(\mu c^{-1} d\right)\right)= \begin{cases}m & \text { if } c^{-1} d \in \frac{1}{2} \mathbb{Z}+B^{(0)} \\ 0 & \text { otherwise. }\end{cases}
$$

If $I$ is a left $R$-ideal with $n(I) \mathbb{Z}=\mathbb{Z}$ and $c \in I^{-1}=\bar{I}$ with $c \neq 0, u \in \widehat{R} \cap B^{(0)}$, then

$$
\begin{aligned}
\sum_{d \in c B^{(0)} \cap \bar{I} / c R^{(0)}} \exp \left(2 \pi i \operatorname{tr}\left(\bar{u} c^{-1} d\right)\right) & \\
= & n(c)^{-1} \sum_{d \in \bar{I} / c R} \exp \left(2 \pi i \operatorname{tr}\left(\bar{u} c^{-1} d\right)\right) \sum_{\mu \in \mathbb{Z} / n(c) \mathbb{Z}} \exp \left(2 \pi i \operatorname{tr}\left(\mu c^{-1} d\right)\right) .
\end{aligned}
$$

If $u \in \widehat{R^{(0)}}$ and $u \notin \widehat{R}$ the same equality holds with $u$ replaced by $u+1 / 2$ on the right hand side of the identity.

Proof. The first part of our assertion is obvious. From it we deduce in the case $u \in \widehat{R} \cap B^{(0)}$ that the right hand side of the second identity is equal to

$$
\sum_{d \in c\left(\frac{1}{2} \mathbb{Z}+B^{(0)}\right) \cap \bar{I} / c R} \exp \left(2 \pi i \operatorname{tr}\left(\bar{u} c^{-1} d\right)\right)
$$

Since $R$ always contains elements of trace 1 , any $d$ satisfying the summation condition in this sum can be changed modulo $c R$ to an element of $c B^{(0)} \cap \bar{I}$. If $u \in \widehat{R^{(0)}}$ and $u \notin \widehat{R}$, then $u+1 / 2$ is in $\widehat{R}$. This is easily checked by examining the completions at the prime 2: If $B$ is ramified at 2, then in the usual basis $\{1, i, j, k\}$ of the Hamilton quaternions, $\left(\widehat{R^{(0)}}\right)_{2}$ is the set of 
elements in $B_{2}^{(0)}$ with half integral coordinates and $\widehat{R}_{2}$ is the set of elements in $B_{2}$ with half integral coordinates whose sum of the coordinates is integral. If $B$ is split at 2 then $\widehat{R}_{2}=R_{2}$ is the ring of $2 \times 2$ matrices over $\mathbb{Z}_{2}$ and $\left(\widehat{R^{(0)}}\right)_{2}$ consists of the matrices of trace 0 that are integral off the diagonal and have half integral entries with integral sum on the diagonal. In either case we see $\left(\widehat{R^{(0)}}\right)_{2}=B(0)_{2} \cap\left(\frac{1}{2} \mathbb{Z}_{2}+\widehat{R}_{2}\right)$. Since replacing $u$ by $u+1 / 2$ does not change the left hand side of the second identity of the lemma, we obtain the assertion.

Lemma 2.3. Let $u \in \widehat{R}$, let $I$ be a left $R$-ideal with $n(I) \mathbb{Z}=\mathbb{Z}$, and let $c \in \bar{I}$ with $c \neq 0$. Then

$$
\sum_{d \in \bar{I} / c R} \exp \left(2 \pi i \operatorname{tr}\left(u c^{-1} d\right)\right)= \begin{cases}n(c)^{2} & \text { if } u c^{-1} \in \widehat{R} I, \\ 0 & \text { otherwise. }\end{cases}
$$

Pr o of. Clear.

We are now ready to formulate

Proposition 2.4. For $u \in \widehat{R^{(0)}}$ one has

$$
b_{u}^{(i)}(s)=\left|R_{i}^{\times}\right| \sum_{m=1}^{\infty} m^{1-s} \sum_{\substack{\mu \in \frac{1}{2} \mathbb{Z} / m \mathbb{Z} \\ \mu^{2}+n(u) \in D^{-1} m \mathbb{Z}}} N\left(\mu, I_{i}, u, m\right),
$$

where $N\left(\mu, I_{i}, u, m\right)$ is the number of left $R$-ideals $J$ in the class of $\widehat{R} I_{i}$ with $\widehat{R} \supseteq J \supseteq(\mu+\bar{u})$ and $n(J)=m D^{-1} \mathbb{Z}$.

Proof. From the definition of $b_{u}^{(i)}(s)$ it is obvious that elements $c \in I_{i}^{-1}$ generating the same left $R_{i}$-ideal give the same contribution, so

$$
b_{u}^{(i)}(s)=\left|R_{i}^{\times}\right| \sum_{m=1}^{\infty} m^{-s} \sum_{\begin{array}{c}
R_{i} c \subset I_{i}^{-1} \\
n\left(R_{i} c\right)=m \mathbb{Z}
\end{array}} \sum_{d \in c B^{(0)} \cap I_{i}^{-1} / c R^{(0)}} \exp \left(2 \pi i \operatorname{tr}\left(\bar{u} c^{-1} d\right)\right) .
$$

The innermost sum is then transformed using Lemmas 2.2 and 2.3 to give $m \sum_{\mu \in \frac{1}{2} \mathbb{Z} / m \mathbb{Z}} 1$, where the summation runs over $\mu$ satisfying $u+\mu \in \widehat{R}$, $\mu-u \in \widehat{R} I_{i} c$. One sees that all $\mu$ in the sum have to satisfy $\mu^{2}+n(u) \in$ $D^{-1} m \mathbb{Z}$ and that this condition already implies $u+\mu \in \widehat{R}$ (using again the explicit description of $\widehat{R^{(0)}}$ and of $\widehat{R}$ in the proof of Lemma 2.2). We can then change the order of summation to get

$$
b_{u}^{(i)}(s)=\left|R_{i}^{\times}\right| \sum_{m=1}^{\infty} m^{1-s} \sum_{\substack{\mu \in \frac{1}{2} \mathbb{Z} / m \mathbb{Z} \\ \mu^{2}+n(u) \in D^{-1} m \mathbb{Z}}} N\left(\mu, I_{i}, u, m\right)
$$


as asserted, putting $J(c)=J=\widehat{R} I_{i} c$ for each ideal $R_{i} c$ in the original sum.

The following lemma implies that we get a smoother expression if we sum the $b_{u}^{(i)}(s)$ for $i=1, \ldots, h$ weighted with factors $\left|R_{i}^{\times}\right|^{-1}$ :

LeMma 2.5. Let $\mu \in \frac{1}{2} \mathbb{Z}$ satisfy $\mu^{2}+n(u) \in D^{-1} m \mathbb{Z}$, denote by $\widetilde{\sigma}(m, u, \mu)$ the sum of all $t \mid m$ satisfying $\operatorname{gcd}(t, D)=1, \mu+u \in t \widehat{R}, t \mid\left(\mu^{2}+n(u)\right) D / m$. Then

$$
\sum_{i=1}^{h} N\left(\mu, I_{i}, u, m\right)=\widetilde{\sigma}(m, u, \mu) .
$$

Proof. Upon summation of the $N\left(\mu, I_{i}, u, m\right)$ over $i$ the restriction on the class of the ideal $J$ in their definition is omitted and it is clear that

$$
\sum_{i=1}^{h} N\left(\mu, I_{i}, u, m\right)=\prod_{p} N_{p}(\mu, u, m),
$$

where $N_{p}(\mu, u, m)$ is the number of left $R_{p}$-ideals $J_{p}$ of norm $m D^{-1} \mathbb{Z}_{p}$ between $\widehat{R}_{p}$ and $R_{p}(\mu+\bar{u})$. For $p \mid D$ there is just one $R_{p}$-ideal of given norm and this ideal contains all ideals whose norm is divisible by its norm, so $N_{p}(\mu, u, m)=1$ for all $\mu$ with $\mu^{2}+n(u) \in D^{-1} m \mathbb{Z}$. For $p \nmid D$ we have $\widehat{R}_{p}=R_{p}$ and $D \in \mathbb{Z}_{p}^{\times}$. If we write $\bar{u}+\mu=p^{r} v$ with $v \in R_{p}, v \notin p R_{p}$ and $n(v) \in p^{\kappa} \mathbb{Z}_{p}^{\times}$and let $m \mathbb{Z}_{p}=p^{\nu} \mathbb{Z}_{p}$, then our assertion is equivalent to

$$
N_{p}(\mu, u, m)=\sum_{\lambda \leq \min (\nu, \kappa+2 r-\nu, r)} p^{\lambda} .
$$

Since $N_{p}(\mu, u, m)$ depends only on $\nu, \kappa$, and $r$, we write $N_{p}(\nu, \kappa, r):=$ $N_{p}(\mu, u, m)$ and define $N_{p, 0}(\nu, \kappa, r)$ by $N_{p}(\nu, \kappa, r)=N_{p, 0}(\nu, \kappa, r)+N_{p}(\nu-$ $2, \kappa, r-1)$, so that $N_{p, 0}(\nu, \kappa, r)$ is just the number of left $R_{p}$-ideals of norm $p^{\nu} \mathbb{Z}_{p}$ between $R_{p}$ and $R_{p}(\mu+u)$ that are not divisible by $p$. It is well known that the left $R_{p}$-ideals in $R_{p}$ that are not divisible by $p$ are in one-to-one correspondence with the vertices in the "tree of $\mathrm{SL}_{2}\left(\mathbb{Q}_{p}\right)$ " (i.e., the Bruhat-Tits building of this group) [Vi], where the $p$-adic valuation of the norm of the ideal is equal to the distance from the vertex $R_{p}$ in that tree. From this one sees easily that

$$
N_{p, 0}(\nu, \kappa, r)= \begin{cases}p^{\nu}+p^{\nu-1} & \text { if } \nu \leq r \\ p^{r} & \text { if } r<\nu \leq \kappa+r \\ 0 & \text { if } \nu>\kappa+r\end{cases}
$$

which by induction implies our assertion.

In what follows the crucial role is played by the Cohen-Zagier $L$-function $L(s, \Delta)$ (see [Za1], p. 130). For any integral $\Delta$ the function $L(s, \Delta)$ is defined 
by the equalities

$$
\frac{\zeta(s) L(s, \Delta)}{\zeta(2 s)}=\sum_{m=1}^{\infty} a(m, \Delta) m^{-s},
$$

where

$$
a(m, \Delta)=\#\left\{b \bmod 2 m \mid b^{2} \equiv \Delta \bmod 4 m\right\} .
$$

We shall enumerate some properties of $L(s, \Delta)$ :

$$
\begin{aligned}
& L(s, \Delta)=0 \quad \text { if } \Delta \text { is not a discriminant }(\Delta \equiv 2,3 \bmod 4), \\
& L(s, 1)=\zeta(s), \quad L(s, 0)=\zeta(2 s-1), \\
& L(s, \Delta)=L\left(s,\left(\frac{\Delta}{*}\right)\right) \quad \text { if } \Delta \text { is a fundamental discriminant, } \\
& L(s, \Delta)=L\left(s,\left(\frac{\Delta_{0}}{*}\right)\right) \sum_{t \mid f} \mu(t)\left(\frac{\Delta_{0}}{t}\right) t^{-s} \sigma_{1-2 s}\left(\frac{f}{t}\right) \quad \text { if } \Delta \equiv 0,1 \bmod 4 ;
\end{aligned}
$$

$\Delta=\Delta_{0} f^{2}$ with natural $f ; \Delta_{0}$ is the discriminant of the quadratic field $\mathbb{Q}(\sqrt{\Delta}),\left(\frac{\Delta_{0}}{*}\right)$ is the Kronecker symbol;

$$
L\left(s,\left(\frac{\Delta_{0}}{*}\right)\right)=\sum_{m \geq 1}\left(\frac{\Delta_{0}}{n}\right) n^{-s}
$$

the associated $L$-series, and $\sigma_{\nu}(m)=\sum_{t \mid m} t^{\nu}$. The function

$$
L^{*}(s, \Delta)= \begin{cases}\pi^{-s / 2} \Gamma(s / 2) \Delta^{s / 2} L(s, \Delta) & \text { for } \Delta>0 \\ \pi^{-s / 2} \Gamma((s+1) / 2)|\Delta|^{s / 2} L(s, \Delta) & \text { for } \Delta<0\end{cases}
$$

has a meromorphic continuation to the whole complex plane and satisfies the functional equation

$$
L^{*}(s, \Delta)=L^{*}(1-s, \Delta) .
$$

The function $L(s, \Delta)$ has no poles if $\Delta$ is negative.

The values of the functions $L(s,-N)(N>0)$ at even negative points are rational. These are the so-called Cohen numbers (see $[\mathrm{Co}]$ ):

$$
L(-2 k,-N)=H(2 k+1, N) .
$$

In particular, $H(1, N)=H(N)$ is the class number of $\mathrm{SL}_{2}$-non-equivalent binary quadratic forms of discriminant $-N$, calculated with multiplicity $1 / 2$ (or $1 / 6,1 / 4$ ) if the discriminant of the form is less than -4 (equal to $-3,-4$ ) (see [Za1], p. 113). 
THEOREM 2.6. For $u \in \widehat{R^{(0)}}$ and $u \neq 0$ one has

$$
\begin{aligned}
\widetilde{b}_{u}(s)=\sum_{i=1}^{h} \frac{b_{u}^{(i)}(s)}{\left|R_{i}^{\times}\right|}= & \zeta(s-1) \zeta(2 s-2)^{-1} \prod_{p \mid D}\left(1+p^{1-s}\right)^{-1} \\
& \times \sum_{\substack{t \mid u \\
(t, D)=1}} \sum_{l \mid D q(2 u)^{-1}}(t l)^{2-s} L\left(s-1,-\frac{n(2 u) D^{2}}{(t l)^{2}}\right),
\end{aligned}
$$

where the product is taken over all prime divisors of the discriminant $D$; the sum over $t$ runs over $t \in \mathbb{N}$ relatively prime to $D$ satisfying $u \in t \widehat{R^{(0)}} ; q(u)$ denotes the denominator of $n(u)$ (the quaternion norm of $u$ ); the second sum is taken over all positive divisors $l$ of the number $D / q(2 u)$ and $L(\ldots)$ is the L-function defined in (2.1).

For $u=0$ one has

$$
\widetilde{b}_{0}(s)=\zeta(s-1) \zeta(s-2) \zeta(2 s-3) \zeta(2 s-2)^{-1} \prod_{p \mid D} \frac{1-p^{4-2 s}}{1+p^{1-s}} .
$$

Proof. For $0 \neq u \in \widehat{R^{(0)}}$ we first prove the following formula:

$$
\widetilde{b}_{u}(s):=\sum_{i=1}^{h} \frac{b_{u}^{(i)}(s)}{\left|R_{i}^{\times}\right|}=\sum_{t} t^{2-s} \sum_{m=1}^{\infty} m^{1-s} a\left(D m, \frac{-4 n(u) D^{2}}{t^{2}}\right),
$$

where the sum over $t$ runs over the natural numbers $t$ which are coprime to $D$ and for which $u \in t \widehat{R^{(0)}}$.

By Lemma 2.5 we have

$$
\widetilde{b}_{u}(s)=\sum_{m=1}^{\infty} m^{1-s} \sum_{\mu} \sum_{t} t
$$

where $\mu$ runs over $\mu \in \frac{1}{2} \mathbb{Z} / m \mathbb{Z}$ with $\mu^{2}+n(u) \in D^{-1} m \mathbb{Z}$ and $t$ runs over $t \mid m$ with $\mu+u \in t \widehat{R}, t \mid\left(\mu^{2}+n(u)\right) D / m$, and $\operatorname{gcd}(t, D)=1$. Collecting all terms with the same $t$ and replacing $\mu$ by $\mu / t, m$ by $m / t$, we obtain the assertion with $a\left(D m,-4 n(u) D^{2} / t^{2}\right)$ replaced by $\#\left\{\mu \in \frac{1}{2} \mathbb{Z} / m \mathbb{Z} \mid \mu^{2}+\right.$ $\left.n(u) / t^{2} \in D^{-1} m \mathbb{Z}\right\}$. This is obviously equal to

$$
\begin{aligned}
\#\left\{\mu \in \mathbb{Z} / 2 m \mathbb{Z} \mid \mu^{2}+4 n(u) / t^{2} \in 4 D^{-1} m \mathbb{Z}\right\} \\
=\#\left\{\mu \in \mathbb{Z} / 2 D m \mathbb{Z} \mid \mu^{2}+4 n(u) D^{2} / t^{2} \in 4 D m \mathbb{Z}\right\},
\end{aligned}
$$

the last equality being obtained by replacing $\mu$ by $D \mu$ and observing that $\mu^{2}+4 n(u) D^{2} / t^{2} \in 4 D m \mathbb{Z}$ implies $D \mid \mu$. This proves formula (2.3). 
Denote the main part of the coefficient $\widetilde{b}_{u}(s)$ by

$$
S_{D}(N, q ; s)=\sum_{m \geq 1} a\left(m D,-\frac{N D^{2}}{q}\right) m^{-s} .
$$

Lemma 2.7. Assume that $D$ is a square-free natural number; $q$ is an odd divisor of $D$ and $N$ is a natural number relatively prime to $q$. Then

$$
S_{D}(N, q ; s)=\zeta(s) \zeta(2 s)^{-1} \prod_{p \mid D}\left(1+p^{-s}\right)^{-1} \sum_{l \mid \frac{D}{q}} l^{1-s} L\left(s,-\frac{N D^{2}}{q l^{2}}\right) .
$$

In particular, if $N=0$ and $q=1$ then

$$
S_{D}(0,1 ; s)=\zeta(s) \zeta(2 s-1) \zeta(2 s)^{-1} \prod_{p \mid D} \frac{1+p^{1-s}}{1+p^{-s}} .
$$

Proof. Without loss of generality we may assume that the number $-N D^{2} / q$ is a discriminant, i.e. $-N D^{2} / q \equiv 0,1(\bmod 4)$. We shall express the Dirichlet series $S_{D}(N, q ; s)$ as the product of three factors

$$
\begin{aligned}
S_{D}(N, q ; s)= & \left(\sum_{(m, D)=1} a\left(m D,-q^{-1} N D^{2}\right) m^{-s}\right) \\
& \times \prod_{p \mid q}\left(\sum_{\delta \geq 0} a\left(p^{\delta+1},-q^{-1} N D^{2}\right) p^{-\delta s}\right) \\
& \times \prod_{p \mid \frac{D}{q}}\left(\sum_{\delta \geq 0} a\left(p^{\delta+1},-q^{-1} N D^{2}\right) p^{-\delta s}\right) .
\end{aligned}
$$

By definition of the $L$-function $(2.1)$ and by the fact that $a\left(D,-q^{-1} N D^{2}\right)$ $=1$ for the square-free $D$ we see that the first factor in the last product is equal to

$$
\zeta^{(D)}(s) \zeta^{(D)}(2 s)^{-1} L^{(D)}\left(s,-\frac{N D^{2}}{q}\right),
$$

where $\zeta^{(D)}$ and $L^{(D)}$ are Euler products without prime divisors of $D$.

If we denote by $D_{0}$ the discriminant of the quadratic field $\mathbb{Q}\left(\sqrt{-q^{-1} N D^{2}}\right)$, then $-N D^{2} / q=D_{0} f^{2}$, and $\nu_{p}=\operatorname{ord}_{p} f$ is the $p$-order of $f$. It follows from the properties of the function $L(s, \Delta)$ that its $p$-local factors are of the following form:

$$
L_{p}\left(s,-\frac{N D^{2}}{q}\right)=\left(1-\left(\frac{\Delta_{0}}{p}\right) p^{-s}\right)^{-1} F_{p}\left(s, \nu_{p}\right),
$$

where

$$
F_{p}\left(s, \nu_{p}\right)=\sigma_{1-2 s}\left(p^{\nu_{p}}\right)-\left(\frac{\Delta_{0}}{p}\right) p^{-s} \sigma_{1-2 s}\left(p^{\nu_{p}-1}\right) .
$$


Under the hypotheses of the lemma, $q$ is an odd divisor of $D_{0}$, so the second factor in (2.4) is equal to

$$
\prod_{p \mid q} p^{s}\left(1+p^{-s}\right)\left(L_{p}\left(s,-\frac{N D^{2}}{q}\right)-1\right)=1 .
$$

If $p$ is a divisor of $D / q$, then any local factor in the third product in (2.4) is equal to

$$
p^{s}\left(\sum_{\delta \geq 0} a\left(p^{\delta},-\frac{N D^{2}}{q}\right) p^{-\delta s}-1\right)=\frac{F_{p}\left(s, \nu_{p}\right)+p^{1-s} F_{p}\left(s, \nu_{p}-1\right)}{\left(1-\left(\frac{\Delta_{0}}{p}\right) p^{-s}\right)},
$$

because $\sigma_{s}\left(p^{\nu}\right)-1=p^{s} \sigma_{s}\left(p^{\nu-1}\right)$.

The terms $F_{p}\left(s, \nu_{p}\right)$ give us the factors $L_{p}\left(s,-q^{-1} N D^{2}\right)$ in (2.4) and the terms with $F_{p}\left(s, \nu_{p}-1\right)$ give the factors $p^{1-s} L_{p}\left(s,-q^{-1} p^{-2} N D^{2}\right)$. Collecting all factors in (2.4) we get the formula of the lemma.

Now we can finish the proof of Theorem 2.6.

By the identity (2.3) and the formula of the last lemma we have

$$
\begin{aligned}
\widetilde{b}_{u}=\zeta(s-1) \zeta(2 s-2)^{-1} & \prod_{p \mid D}\left(1+p^{1-s}\right)^{-1} \\
& \times \sum_{\substack{t \mid u \\
(t, D)=1}} \sum_{\frac{D}{q\left(2 u t^{-1}\right)}}(t l)^{2-s} L\left(s-1,-\frac{n(2 u) D^{2}}{(t l)^{2}}\right),
\end{aligned}
$$

where $q$ is the denominator of the norm of the quaternion $2 u t^{-1}$. From the description of the 2-adic completions in the proof of Lemma 2.2 we see that the denominator $q$ of the norm of $2 u$, where $u$ is a quaternion from the lattice $\widehat{R^{(0)}}$, is an odd number. Since obviously $q\left(2 u t^{-1}\right)=q(2 u)$ for any natural $t$ coprime to $D$ with $u \in t \widehat{R^{(0)}}$, we obtain the formula of Theorem 2.6 for $u \neq 0$. If $u=0$, then the summation in (2.3) is taken over all natural $t$ coprime to $D, q(u)=1$ and $L(s-1,0)=\zeta(2 s-3)$. Theorem 2.6 is proved.

We shall now add an additional factor to the Eisenstein series so that it satisfies a functional equation. Let us define the series

$E_{R}^{*}(z, s)=\pi^{-3 s / 2+1} D^{s} \Gamma(s / 2) \Gamma(s) \zeta(2 s-2) \zeta(s-1)^{-1} \prod_{p \mid D}\left(1+p^{1-s}\right) \widetilde{E}_{R}(z, s)$,

where the product is taken over all prime divisors of the discriminant $D$ and

$$
\widetilde{E}_{R}(z, s)=\sum_{i=1}^{h} \frac{1}{\left|R_{i}^{\times}\right|} \widetilde{E}_{i}(z, s) .
$$

As a corollary of Theorem 2.6 we can prove the following 
Theorem 2.8. The Eisenstein series $E_{R}^{*}(z, s)$ has the following Fourier expansion at infinity:

$$
\begin{aligned}
& E_{R}^{*}(z, s)= z_{r}^{s} f_{0}^{(D)}(s)+z_{r}^{3-s} f_{0}^{(D)}(3-s) \\
&+4 \sqrt{\pi} \sum_{\substack{u \in R^{(0)} \\
u \neq 0}} n(2 u)^{-1 / 4} \sum_{\substack{t \mid u \\
(t, D)=1}} \sum_{l \mid \frac{D}{q(2 u)}} t L^{*}\left(s-1,-\frac{n(2 u) D^{2}}{t^{2} l^{2}}\right) \\
& \quad \times z_{r}^{3 / 2} K_{s-3 / 2}\left(2 \pi z_{r} \sqrt{n(2 u)}\right) \exp (2 \pi i \operatorname{tr}(\bar{u} z)),
\end{aligned}
$$

where

$$
f_{0}^{(D)}(s)=(s-1) D^{s} \prod_{p \mid D}\left(1-p^{2-2 s}\right) \zeta^{*}(s) \zeta^{*}(2 s-2),
$$

the function $L^{*}$ is defined in (2.1), (2.2) and, as is customary, $\zeta^{*}(s)=$ $\pi^{-s / 2} \Gamma(s / 2) \zeta(s)$.

R e mark 2.9. One should also get nice formulae for Fourier coefficients of other linear combinations of the Eisenstein series $\widetilde{E}_{i}(z, s)$, in particular for linear combinations $\sum_{i=1}^{h} \frac{\alpha_{i}}{\left|R_{i}^{\times}\right|} \widetilde{E}_{i}(z, s)$ whose coefficients $\alpha_{i}$ are the values of an eigenfunction of all Hecke operators on the adelization $B_{\mathbb{A}}^{\times}$ (see [BS]). We plan to come back to such linear combinations of Eisenstein series as part of a more general investigation into the theta correspondence between the unitary groups of (skew-) hermitian forms over quaternion algebras.

The next result is a generalization of the theorem proved in [Gr1] for $D=2$ (see also [Gr2], Lemma 3.2).

Corollary 2.10. The Eisenstein series $E_{R}^{*}(z, s)$ has a meromorphic continuation to the whole plane and satisfies the functional equation

$$
E_{R}^{*}(z, s)=E_{R}^{*}(z, 3-s) .
$$

It is entire except for simple poles at $s=0,3$, with the residue

$$
\frac{\zeta(3)}{2 \pi} \prod_{p \mid D}\left(p^{2}-1\right)
$$

at the point $s=3$.

Proof. We only have to recall that

$$
K_{s}(y)=K_{-s}(y), \quad L^{*}(s-1, \Delta)=L^{*}(2-s, \Delta)
$$

and that the $L$-function $L(s, \Delta)$ has no poles when $\Delta<0$. 
Proof of Theorem 2.8. According to Lemma 2.1 and Theorem 2.6 the zeroth Fourier coefficient of the series $E_{R}^{*}(z, s)$ is equal to

$$
\begin{aligned}
& F(s)\left(z_{r}^{s} \sum_{i} \frac{Z\left(I_{i}, s\right)}{\left|R_{i}^{\times}\right|}\right. \\
+ & \left.\frac{2}{D} z_{r}^{3-s} \pi^{3 / 2} \Gamma\left(s-\frac{3}{2}\right) \Gamma(s)^{-1} \frac{\zeta(s-1) \zeta(s-2) \zeta(2 s-3)}{\zeta(2 s-2)} \prod_{p \mid D} \frac{1-p^{4-2 s}}{1+p^{1-s}}\right),
\end{aligned}
$$

where

$$
F(s)=\pi^{-3 s / 2+1} D^{s} \Gamma(s / 2) \Gamma(s) \zeta(2 s-2) \zeta(s-1)^{-1} \prod_{p \mid D}\left(1+p^{1-s}\right)
$$

is the factor of holomorphy of the Eisenstein series. The first summand in $(2.5)$ is equal to $z_{r}^{s} f_{0}^{(D)}(s)$, because

$$
\sum_{i} \frac{Z\left(I_{i}, s\right)}{\left|R_{i}^{\times}\right|}=\zeta(s) \zeta(s-1) \prod_{p \mid D}\left(1-p^{1-s}\right) .
$$

(The left hand side is $\sum_{m} A_{m} m^{-s}$, where $A_{m}$ is the number of left $R$-ideals of reduced norm $m$. The asserted identity therefore follows from the results of [L], see also [Vi], III.2.)

The second summand in (2.5) is equal to

$$
z_{r}^{3-s}(s-2) \zeta^{*}(s-2) \zeta^{*}(2 s-3) D^{s-1} \prod_{p \mid D}\left(1-p^{4-2 s}\right)=z_{r}^{3-s} f_{0}^{(D)}(3-s),
$$

since $D$ has an odd number of prime divisors. The theorem is proved.

EXAMPLE 2.11. Let us take the quaternion algebra with discriminant $D=2$ and the unique type of maximal order $R$ in it:

$$
\begin{gathered}
B=\left(\frac{-1,-1}{\mathbb{Q}}\right)=\mathbb{Q} \oplus \mathbb{Q} i \oplus \mathbb{Q} j \oplus \mathbb{Q} k, \\
R=\left\{\frac{a+b i+c j+d k}{2} \mid a \equiv b \equiv c \equiv d \bmod 2\right\},
\end{gathered}
$$

where $i^{2}=j^{2}=k^{2}=-1$. By Theorem 2.8,

$$
\begin{aligned}
E_{R}^{*}(z, s)= & z_{r}^{s} f_{0}^{(2)}(s)+z_{r}^{3-s} f_{0}^{(2)}(3-s) \\
& +4 \sqrt{\pi} \sum_{\substack{u=(a, b, c) \neq 0 \\
a, b, c \in \mathbb{Z}}} n(u)^{-1 / 4} \\
& \times \sum_{\substack{t \mid(a, b, c) \\
(t, 2)=1}}\left(t L^{*}\left(s-1,-\frac{4 n(u)}{t^{2}}\right)+2 t L^{*}\left(s-1,-\frac{n(u)}{t^{2}}\right)\right) \\
& \times z_{r}^{3 / 2} K_{s-3 / 2}\left(2 \pi \sqrt{n(u)} z_{r}\right) \exp \left(2 \pi i\left(a z_{1}+b z_{2}+c z_{3}\right)\right),
\end{aligned}
$$


where

$$
z=z_{r}+z_{1} i+z_{2} j+z_{3} k, \quad f_{0}^{(2)}(s)=(s-1) 2^{s}\left(1-2^{2-2 s}\right) \zeta^{*}(s) \zeta^{*}(2 s-2) .
$$

If we restrict the Eisenstein series to the positive axis $z_{r}>0$, then we get the following asymptotics for the sum of the Fourier coefficients:

Corollary 2.12. As $z_{r} \rightarrow 0$ with $s \neq 0,3$ we have

$$
\begin{array}{r}
4 \sqrt{\pi} \sum_{\substack{u \in \widehat{R}(0) \\
u \neq 0}} n(2 u)^{-1 / 4} \sum_{\substack{t \mid u \\
(t, D)=1}} \sum_{\mid \frac{D}{q(2 u)}} t l L^{*}\left(s+\frac{1}{2},-\frac{n(2 u) D^{2}}{t^{2} l^{2}}\right) K_{s}\left(2 \pi z_{r} \sqrt{n(2 u)}\right) \\
=z_{r}^{-s-3} f_{0}^{(D)}\left(\frac{3}{2}+s\right)+z_{r}^{-s+3} f_{0}^{(D)}\left(\frac{3}{2}-s\right)+O\left(z_{r}^{s}\right)+O\left(z_{r}^{-s}\right) .
\end{array}
$$

In accordance with the Tauberian theorem we have the following result for $s=1 / 2$ :

$$
\sum_{\substack{u \in \widehat{R^{(0)}} \\ 0<n(2 u) \leq X}} \sum_{\substack{t \mid u \\(t, D)=1}} \sum_{l \mid \frac{D}{\frac{D}{q(2 u)}}} \frac{t l H\left(\frac{n(2 u) D^{2}}{t^{2} l^{2}}\right)}{\sqrt{n(2 u)}} \sim X^{3 / 2} \frac{\pi^{4}}{54} \prod_{p \mid D}\left(p^{2}-1\right) .
$$

In particular, in the case of Example 2.11 we have

$$
\begin{aligned}
\sum_{n \leq X} \sum_{\substack{a, b, c \in \mathbb{Z} \\
a^{2}+b^{2}+c^{2}=n}} & \sum_{\substack{t \mid(a, b, c) \\
(t, 2)=1}} \frac{t H\left(4 n / t^{2}\right)+2 t H\left(n / t^{2}\right)}{\sqrt{n}} \\
& =\sum_{1 \leq N \leq X} \operatorname{oddsqu}(N, X) \frac{r_{3}(N)(H(4 N)+2 H(N))}{\sqrt{N}} \sim X^{3 / 2} \frac{\pi^{4}}{18},
\end{aligned}
$$

where

$$
\operatorname{oddsqu}(N, X)=\left\lceil\frac{1}{2}\lfloor\sqrt{X / N}\rfloor\right\rceil
$$

is the number of odd squares less than or equal to $X / N$.

We note that the class number $H(N)$ and the number of representations of $N$ as a sum of three squares are connected by the classical formulae

$$
\begin{aligned}
r_{3}(N) & = \begin{cases}12 H(N) & \text { if } N \equiv 1,2 \bmod 4, \\
24 H(N) & \text { if } N \equiv 3 \bmod 8, \\
0 & \text { if } N \equiv 7 \bmod 8,\end{cases} \\
r_{3}(4 N) & =r_{3}(N) .
\end{aligned}
$$

3. Mellin transform. Our next aim is to calculate the Mellin transform of the restriction of the Eisenstein series $E^{*}(s, t)$ to the positive real axis. 
Theorem 3.1. Define the Dirichlet series

$$
\mathcal{D}_{D}(s, t)=\sum_{\substack{m>0 \\ D m \in \mathbb{N}}} \frac{\left.r \widehat{R^{(0)}}(m) \sum_{l}\right|_{\frac{D}{q(m)}} l L^{*}\left(s,-m D^{2} / l^{2}\right)}{m^{t}},
$$

where

$$
r \widehat{R^{(0)}}(m)=\#\left\{u \in \widehat{R^{(0)}} \mid n(2 u)=m\right\}
$$

is the number of representations of $m$ as a norm of an element $2 u$ with $u \in \widehat{R^{(0)}}, q(m)$ is the denominator of $m, L(s, \Delta)$ is the Cohen-Zagier $L$ function defined in (2.1) and

$$
\zeta^{(D)}(s)=\prod_{(p, D)=1}\left(1-p^{-s}\right)^{-1}
$$

is the zeta-function without $D$-part for the discriminant $D$ of the quaternion algebra. The Dirichlet series

$$
\mathcal{D}_{D}^{*}(s, t)=\pi^{-2 t+1} \Gamma\left(t+\frac{s-1}{2}\right) \Gamma\left(t-\frac{s}{2}\right) \zeta^{(D)}(2 t-1) \mathcal{D}_{D}(s, t)
$$

has a meromorphic continuation to the whole complex $s$-and t-planes. It is invariant with respect to the substitution

$$
t \rightarrow 2-t, \quad s \rightarrow 1-s
$$

and could have simple poles with respect to $t$ only for

Moreover,

$$
t=\frac{s}{2}, \quad t=\frac{1-s}{2}, \quad t=\frac{s+3}{2}, \quad t=\frac{4-s}{2} .
$$

$$
\mathcal{D}_{D}^{*}(s, t)+\frac{f_{0}^{(D)}(s+1)}{s+2 t-1}+\frac{f_{0}^{(D)}(s+1)}{s-2 t+3}+\frac{f_{0}^{(D)}(2-s)}{2 t-s}+\frac{f_{0}^{(D)}(2-s)}{4-s-2 t}
$$

is an entire function on the whole complex plane.

Remark 3.2. Mellin transforms of automorphic forms of hyperbolic type were constructed by Maass in [Ma]. Using his method it is possible to add a spherical function of a ternary quadratic form to the Dirichlet series $\mathcal{D}(s, t)$.

R e m a r k 3.3. The only term in the Dirichlet series $\mathcal{D}_{D}(s, t, R)$ from Theorem 3.1 that depends on the choice of the maximal order $R$ in $B$ is the representation number $r_{\widehat{R^{(0)}}}(m)=\#\left\{u \in \widehat{R^{(0)}} \mid n(2 u)=m\right\}$, which obviously depends only on the type of $R$. Furthermore, if $D$ is odd we know from the proof of Lemma 2.2 that the 2-adic completion of $\widehat{R^{(0)}}$ is that of $B^{(0)} \cap\left(\mathbb{Z} \frac{1}{2}+R\right)$, and it is well known that for the odd primes $p \mid D$ the $p$-adic completion of $\widehat{R^{(0)}}$ is $\left\{u \in B_{p}^{(0)} \mid n(u) \in p^{-1} \mathbb{Z}_{p}\right\}$ and that of $R^{(0)}$ is $\left\{u \in B_{p}^{(0)} \mid n(u) \in \mathbb{Z}_{p}\right\}$. 
Hence, putting $L=(\mathbb{Z} 1+2 R) \cap B^{(0)}$ we have

$$
r_{\widehat{R^{(0)}}}(m)=r\left(L, D^{2} m\right),
$$

where $D$ is odd. We let $R$ run through the orders $R_{i}(i=1, \ldots, h)$ and put $L_{i}=\left(\mathbb{Z} 1+2 R_{i}\right) \cap B^{(0)}$. The theta series of the $L_{i}$ have been discussed at some length in [BS]. In particular, we recall from this article that suitable linear combinations of the theta series $\vartheta\left(L_{i}, \tau\right)$ yield the cuspidal newforms $g(\tau)=\sum_{n=1}^{\infty} b_{n} \exp (2 \pi i n \tau)$ of weight $3 / 2$ and level $D$ in Kohnen's space having $b_{n}=0$ for all $n$ with $\left(\frac{-n}{p}\right)=1$ for some $p \mid D$, which correspond under Shimura's correspondence to normalized newforms $f$ of weight 2 with $L(f, 1) \neq 0$. The same linear combinations of the $\mathcal{D}_{D}\left(s, t, R_{i}\right)$ give therefore Dirichlet series in $t$ whose coefficients are formed from such cusp form coefficients and the values at $s$ of Cohen-Zagier's $L$-functions.

Proof of Theorem 3.1. The following formula is well known:

$$
\int_{0}^{\infty} K_{\nu}(a y) y^{t-1} d y=a^{-t} 2^{t-2} \Gamma\left(\frac{t-\nu}{2}\right) \Gamma\left(\frac{t+\nu}{2}\right) \quad \text { for } \operatorname{Re} t>|\operatorname{Re} \nu| .
$$

Let us take the Mellin transformation for $\operatorname{Re} t>\operatorname{Re} s>3$,

$$
\mathbb{M}(s, t)=\int_{0}^{\infty}\left[E^{*}\left(z_{r}, s\right)-f_{0}^{(D)}(s) z_{r}^{s}-f_{0}^{(D)}(3-s) z_{r}^{3-s}\right] z_{r}^{t-1} d z_{r},
$$

and sum firstly over the divisors $d$ in the Dirichlet series. This gives us

$$
\begin{aligned}
\pi^{-t-1} \Gamma\left(\frac{t+s}{2}\right) \Gamma & \left(\frac{t-s+3}{2}\right) \zeta^{(D)}(t+1) \\
& \times \sum_{\substack{u \in \widehat{R}(0) \\
u \neq 0}} n(2 u)^{(t+2) / 2} \sum_{l \mid \frac{D}{q(u)}} L^{*}\left(s-1,-\frac{n(2 u) D^{2}}{l^{2}}\right),
\end{aligned}
$$

where the last Dirichlet series is absolutely convergent for $\operatorname{Re} t>\operatorname{Re} s>3$.

On the other side we can decompose the Mellin integral into a sum of three terms

$$
\begin{aligned}
\mathbb{M}(s, t)= & \int_{1}^{\infty}\left[E^{*}\left(z_{r}, s\right)-f_{0}^{(D)}(s) z_{r}^{s}-f_{0}^{(D)}(3-s) z_{r}^{3-s}\right] z_{r}^{t-1} d z_{r} \\
& +\int_{0}^{1}\left[E^{*}\left(z_{r}, s\right)-f_{0}^{(D)}(s) z_{r}^{-s}-f_{0}^{(D)}(3-s) z_{r}^{s-3}\right] z_{r}^{t-1} d z_{r} \\
& +\int_{0}^{1}\left[f_{0}^{(D)}(s) z_{r}^{-s}-f_{0}^{(D)}(3-s) z_{r}^{s-3}-f_{0}^{(D)}(s) z_{r}^{s}\right.
\end{aligned}
$$




$$
\left.-f_{0}^{(D)}(3-s) z_{r}^{3-s}\right] z_{r}^{t-1} d z_{r} .
$$

The series $E^{*}\left(z_{r}, s\right)$ is invariant with respect to the transformation $z_{r} \rightarrow z_{r}^{-1}$. After obvious calculations we see that the Mellin transform is equal to

$$
\begin{aligned}
\int_{1}^{\infty}\left[E^{*}\left(z_{r}, s\right)\right. & \left.-f_{0}^{(D)}(s) z_{r}^{s}-f_{0}^{(D)}(3-s) z_{r}^{3-s}\right] z_{r}^{t-1} d z_{r} \\
& +\int_{1}^{\infty}\left[E^{*}\left(z_{r}, s\right)-f_{0}^{(D)}(s) z_{r}^{s}-f_{0}^{(D)}(3-s) z_{r}^{3-s}\right] z_{r}^{-t-1} d z_{r} \\
& -\frac{f_{0}^{(D)}(s)}{s+t}-\frac{f_{0}^{(D)}(s)}{s-t}-\frac{f_{0}^{(D)}(3-s)}{3+t-s}-\frac{f_{0}^{(D)}(3-s)}{3-s-t} .
\end{aligned}
$$

The function $\left[E^{*}\left(z_{r}, s\right)-f_{0}^{(D)}(s) z_{r}^{s}-f_{0}^{(D)}(3-s) z_{r}^{3-s}\right]$ has no poles and it decreases exponentially at infinity so the last two integrals exist for any complex $t$. This gives us a meromorphic continuation of the Mellin integral to the whole complex plane. Let us change the variables $s \rightarrow s+1$ and $t \rightarrow 2 t-2$. We then see that the Dirichlet series

$$
\begin{aligned}
\pi^{-2 t+1} & \Gamma\left(t+\frac{s-1}{2}\right) \Gamma\left(t-\frac{s}{2}\right) \zeta^{(D)}(2 t-1) \\
& \times \sum_{\substack{u \in \widehat{R^{(0)}} \\
u \neq 0}} n(2 u)^{-t} \sum_{l \mid \frac{D}{q(u)}} L^{*}\left(s,-\frac{n(2 u) D^{2}}{l^{2}}\right) \\
& +\frac{f_{0}^{(D)}(s+1)}{s+2 t-1}+\frac{f_{0}^{(D)}(s+1)}{s-2 t+3}+\frac{f_{0}^{(D)}(2-s)}{2 t-s}+\frac{f_{0}^{(D)}(2-s)}{4-s-2 t}
\end{aligned}
$$

is invariant with respect to transformations indicated in the theorem and has no poles. In order to finish the proof of Theorem 3.1 we have to collect together those terms in which the quaternions $2 u$ have the same norm.

EXAmple 3.4. For the quaternion algebra with $D=2$ (see example above) we have the Dirichlet series

$$
\mathcal{D}_{2}(s, t)=\sum_{N \geq 1} \frac{r_{3}(N)\left(L^{*}(s,-4 N)+2 L^{*}(s,-N)\right)}{N^{t}},
$$

where $r_{3}(N)$ denotes the number of representations of $N$ as a sum of three squares. In accordance with Theorem 3.1 the series

$$
\mathcal{D}_{2}^{*}(s, t)=\pi^{-2 t+1} \Gamma\left(t+\frac{s-1}{2}\right) \Gamma\left(t-\frac{s}{2}\right)\left(1-2^{1-2 t}\right) \zeta(2 t-1) \mathcal{D}_{2}(s, t)
$$

is invariant with respect to $t \rightarrow 2-t$. For the special values $s=2 k+1$ we get the Dirichlet series with Cohen's numbers $H(2 k+1, N)$ instead of the 
$L$-functions. The theorem tells us that

$$
\begin{aligned}
\operatorname{Res}_{t=k+2} \sum_{N \geq 1} \frac{r_{3}(N)\left(H(2 k+1,4 N)+2^{2 k+1} H(2 k+1, N)\right)}{N^{t+k}} & \\
= & C_{k} \frac{\zeta(2 k+2) \zeta(4 k+2)}{\zeta(2 k+3)},
\end{aligned}
$$

where

$$
C_{k}=\frac{(-1)^{k} 8(2 k) !\left(2^{4 k+2}-1\right)}{\pi^{2 k}\left(2^{2 k+3}-1\right)} .
$$

In particular, we have for $k=0$,

$$
\operatorname{Res}_{t=2} \sum_{N \geq 1} \frac{r_{3}(N)(H(4 N)+2 H(N))}{N^{t}}=\frac{2 \pi^{4}}{21 \zeta(3)} .
$$

Using the Tauberian theorem we have

$$
\begin{aligned}
& \sum_{N \leq X} r_{3}(N)\left(H(2 k+1,4 N)+2^{2 k+1}\right.H(2 k+1, N)) \\
& \sim X^{2 k+2} C_{k} \frac{\zeta(2 k+2) \zeta(4 k+2)}{(k+2) \zeta(2 k+3)}, \\
& \sum_{N \leq X} r_{3}(N)(H(4 N)+2 H(N)) \sim X^{2} \frac{\pi^{4}}{21 \zeta(3)} .
\end{aligned}
$$

EXAMPLE 3.5. Let us take the quaternion algebra with discriminant $D=3$ :

$$
B=\left(\frac{-1,-3}{\mathbb{Q}}\right)=\mathbb{Q} \oplus \mathbb{Q} i \oplus \mathbb{Q} j \oplus \mathbb{Q} k, \quad \text { where } i^{2}=-1, j^{2}=k^{2}=-3,
$$

and the unique type of maximal order $R$ in it

$$
R=\mathbb{Z} \frac{1+j}{2} \oplus \mathbb{Z} \frac{i+k}{2} \oplus \mathbb{Z} j \oplus \mathbb{Z} k
$$

with

$$
\widehat{R^{(0)}}=\left\{a \frac{i+k}{2}+b \frac{j}{6}+c \frac{k}{3} \mid a, b, c \in \mathbb{Z}\right\} .
$$

For any $u$ in $\widehat{R^{(0)}}$,

$$
n(2 u)=\frac{12 a^{2}+12 a c+4 c^{2}+b^{2}}{3} .
$$

The series in Theorem 3.1 is equal to

$$
\mathcal{D}_{3}(s, t)=\sum_{N \geq 1}\left(\frac{r_{F}(N) L^{*}(s,-3 N)}{(N / 3)^{t}}+\frac{3 r_{F}(3 N) L^{*}(s,-N)}{N^{t}}\right),
$$


where $r_{F}(N)=r\left(12 a^{2}+12 a c+4 c^{2}+b^{2} ; N\right)$ is the number of representations of $N$ by the ternary quadratic form.

4. Pullback. Let $\ell$ be any quaternion without real part, i.e., $\bar{\ell}=-\ell$. Let us define the following two-dimensional subspace $\mathcal{H}_{\ell}$ of the four-dimensional hyperbolic space $\mathcal{H}$ :

$$
\mathcal{H}_{\ell}=\{z=y+\ell x \mid y>0\}
$$

and a subgroup

$$
\mathcal{G}_{\ell}=\left\{\widetilde{g}=\left(\begin{array}{cc}
a & \ell b \\
\ell^{-1} c & d
\end{array}\right) \in G \mid a, b, c, d \in \mathbb{R}\right\}
$$

of the group $G$.

LEMma 4.1. $\mathcal{H}_{\ell}$ is invariant with respect to the action of $\mathcal{G}_{\ell}$.

Proof. We can realize the usual two-dimensional upper half plane $H^{+}$ in the form

$$
H_{\ell}^{+}=\left\{\widetilde{\tau}=x+\frac{\sqrt{n(\ell)}}{\ell} y \mid y>0\right\}
$$

The isomorphism $\phi_{\ell}$ between $H^{+}$and $H_{\ell}^{+}$,

$$
\phi_{\ell}: \tau=x+\sqrt{-1} y \rightarrow \widetilde{\tau}=x+\frac{\sqrt{n(\ell)}}{\ell} y,
$$

commutes with the action of $\mathrm{SL}_{2}$ :

$$
\phi_{\ell}(g\langle\tau\rangle)=g\left\langle\phi_{\ell}(\tau)\right\rangle, \quad g \in \mathrm{SL}_{2}(\mathbb{R}) .
$$

The domain $\mathcal{H}_{\ell}$ is obtained from $H_{\ell}^{+}$by multiplication with the quaternion $\ell: \mathcal{H}_{\ell}=\ell \cdot H_{\ell}^{+}$.

It is easy to see that

$$
\text { if } g=\left(\begin{array}{ll}
a & b \\
c & d
\end{array}\right) \in \mathrm{SL}_{2}(\mathbb{R}), \quad \text { then } \widetilde{g}=\left(\begin{array}{cc}
a & \ell b \\
\ell^{-1} c & d
\end{array}\right) \in \mathcal{G}_{\ell} .
$$

By definition of the actions of the groups on the homogeneous domains we have $\widetilde{g}\langle\ell \widetilde{\tau}\rangle=\ell g\langle\widetilde{\tau}\rangle$ for $\widetilde{\tau} \in H_{\ell}^{+}$and $\widetilde{g} \in \mathcal{G}_{\ell}$. The lemma is proved.

Now we shall define a function $\mathcal{E}(z, s)$ on the two-dimensional upper half plane $H^{+}$to be the restriction of the Eisenstein series $E_{R}^{*}(z, s)$ to $\mathcal{H}_{\ell}$. More exactly,

$$
\mathcal{E}_{\ell}(\tau, s)=E_{R}^{*}\left(\ell \phi_{\ell}(\tau), s\right)=E_{R}^{*}(\ell x+\sqrt{n(\ell)} y, s),
$$

where $\tau=x+\sqrt{-1} y \in H^{+}$.

TheOREM 4.2. Let $\ell$ be a primitive quaternion with trace zero. Then the real analytic function $\mathcal{E}_{\ell}(\tau, s)$ defined above is invariant with respect to the 
subgroup $\Gamma_{0}(n(\ell))$ :

$$
\mathcal{E}_{\ell}(g\langle\tau\rangle, s)=\mathcal{E}_{\ell}(\tau, s) \quad \text { for } g=\left(\begin{array}{ll}
a & b \\
c & d
\end{array}\right) \in \mathrm{SL}_{2}(\mathbb{Z}), c \equiv 0(\bmod n(\ell)),
$$

and is invariant with respect to the involution

$$
J_{n(\ell)}=\left(\begin{array}{cc}
0 & -1 \\
n(\ell) & 0
\end{array}\right) .
$$

The function $\mathcal{E}_{\ell}(\tau, s)$ has the following Fourier expansion at infinity:

$$
\begin{aligned}
\mathcal{E}_{\ell}(\tau, s)= & y^{s} n(\ell)^{s} f_{0}^{(D)}(s)+y^{3-s} n(\ell)^{3-s} f_{0}^{(D)}(3-s) \\
& +4 \sqrt{\pi} \sum_{\substack{u \in R^{(0)} \\
u \neq 0}} n(2 u)^{-1 / 4} \sum_{\substack{t \mid u \\
(t, D)=1}} \sum_{l \mid \frac{D}{q(2 u)}} t l L^{*}\left(s-1,-\frac{n(2 u) D^{2}}{t^{2} l^{2}}\right) \\
& \times(y \sqrt{n(\ell)})^{3 / 2} K_{s-3 / 2}(2 \pi \sqrt{n(2 u \ell)} y) \exp (2 \pi i x \operatorname{tr}(\bar{u} \ell)),
\end{aligned}
$$

where $f_{0}^{(D)}(s), L^{*}(\ldots)$ and the indices of summations are as defined in Theorem 2.8 .

Proof. For an integral matrix $g$ we see that

$$
g=\left(\begin{array}{ll}
a & b \\
c & d
\end{array}\right) \in \Gamma_{0}(n(\ell)) \quad \text { is equivalent to } \quad \widetilde{g}=\left(\begin{array}{cc}
a & \ell b \\
\ell^{-1} c & d
\end{array}\right) \in \Gamma_{R} .
$$

According to the formulae obtained above

$$
\begin{aligned}
\mathcal{E}_{\ell}(g\langle\tau\rangle, s) & =E^{*}(\ell(\widetilde{g\langle\tau\rangle}), s)=E^{*}(\ell(g\langle\widetilde{\tau}\rangle), s) \\
& =E^{*}(\widetilde{g}\langle\ell \widetilde{\tau}\rangle, s)=E^{*}(\ell \widetilde{\tau}, s)=\mathcal{E}_{\ell}(\tau, s),
\end{aligned}
$$

where $\widetilde{\tau}=\phi_{\ell}(\tau)$ for $\tau \in H^{+}$. It is easy to see that

$$
\ell \phi_{\ell}\left(-\frac{1}{n(\ell) \tau}\right)=\left(\ell \phi_{\ell}(\tau)\right)^{-1}
$$

This proves the second statement of the theorem. The form of the Fourier expansion follows from Theorem 2.8.

Remark 4.3. The terms with $y^{s}$ and $y^{3-s}$ in the Fourier expansions depend only on the discriminant of the quaternion algebra $B$ and the norm of quaternion $\ell$, but do not depend on $\ell$ itself or on the maximal order $R$. This gives us a series of examples of functions in $L^{2}\left(\Gamma_{0}(n(\ell)) \backslash H^{+}\right)$. Take two quaternions $\ell_{1} \in R_{1}, \ell_{2} \in R_{2}$ of the same norm $n\left(\ell_{1}\right)=n\left(\ell_{2}\right)=p$, where $p$ is a prime number and $R_{1}$ and $R_{2}$ are maximal orders of the quaternion algebra $H$. The difference

$$
\mathcal{E}_{\ell_{1}}^{R_{1}}(\tau, s)-\mathcal{E}_{\ell_{2}}^{R_{2}}(\tau, s)
$$

belongs to the space $L^{2}\left(\Gamma_{0}(n(\ell)) \backslash H^{+}\right)$. The restriction of this function to the axis $y>0$ is not zero if the Mellin transformations of the Eisenstein 
series $E_{R_{1}}$ and $E_{R_{2}}$, constructed in Theorem 3.1, are not equal. The last fact is easy to check.

EXAMPLE 4.4. Let us take the case of the quaternion algebra with discriminant two. We have the real analytic function $\mathcal{E}_{i}(\tau, s)\left(i^{2}=-1\right)$ with the following Fourier expansion at infinity:

$$
\begin{aligned}
\mathcal{E}_{i}(\tau, s)= & y^{s} f_{0}^{(2)}(s)+y^{3-s} f_{0}^{(2)}(3-s) \\
& +4 \sqrt{\pi} \sum_{\substack{u=n i+b j+c k \\
u \neq 0}} n(u)^{-1 / 4} \sum_{\substack{d \mid(n, b, c) \\
(d, 2)=1}} d\left(L^{*}\left(s-1,-\frac{4 n(u)}{d^{2}}\right)\right. \\
& \left.+2 L^{*}\left(s-1,-\frac{n(u)}{d^{2}}\right)\right) y^{3 / 2} K_{s-3 / 2}(2 \pi \sqrt{n(u)} y) \exp (2 \pi i n x),
\end{aligned}
$$

where

$$
f_{0}^{(2)}(s)=(s-1) 2^{s}\left(1-2^{2-2 s}\right) \zeta^{*}(s) \zeta^{*}(2 s-2) \quad \text { and } \quad n(u)=n^{2}+b^{2}+c^{2} .
$$

It is $\mathrm{SL}_{2}(\mathbb{Z})$-invariant, i.e. $\mathcal{E}_{i}(g\langle\tau\rangle, s)=\mathcal{E}_{i}(\tau, s)$ for $g \in \mathrm{SL}_{2}(\mathbb{Z})$. After resummation we have

$$
\mathcal{E}_{i}(\tau, s)=a_{0}(y, s)+\sum_{n \neq 0} a_{n}(y, s) \exp (2 \pi i n x),
$$

with

$$
\begin{aligned}
a_{0}(y, s)= & y^{s} f_{0}^{(2)}(s)+y^{3-s} f_{0}^{(2)}(3-s) \\
& +8 \sqrt{\pi} \sum_{\substack{m \geq 1 \\
d \geq 1,(2, d)=1}} d^{1 / 2} m^{-1 / 4} r_{2}(m) \\
& \times\left(L^{*}(s-1,-4 m)+2 L^{*}(s-1,-m)\right) y^{3 / 2} K_{s-3 / 2}(2 \pi d \sqrt{m} y)
\end{aligned}
$$

and

$$
\begin{aligned}
a_{n}(y, s)= & 4 \sqrt{\pi} \sum_{\substack{d \mid n \\
(2, d)=1}} d^{1 / 2} \sum_{m \geq 1} m^{-1 / 4} r_{2}\left(m-\frac{n^{2}}{d^{2}}\right) \\
& \times\left(L^{*}(s-1,-4 m)+2 L^{*}(s-1,-m)\right) y^{3 / 2} K_{s-3 / 2}(2 \pi d \sqrt{m} y),
\end{aligned}
$$

where

$$
r_{2}(m)=\#\left\{(b, c) \mid b^{2}+c^{2}=m\right\}
$$

is the number of representations of the natural $m$ as a sum of two squares.

EXAMPLE 4.5. Let us take the algebra with discriminant equal to 3 . We keep the notations of Example 3.5. For the quaternion $j$ we have

$$
n(j)=3, \quad \widehat{R_{j}^{(0)}}=\left\{a \frac{i+k}{2}+c \frac{k}{3} \mid a, c \in \mathbb{Z}\right\} .
$$


The coefficients of the Fourier expansion

$$
\mathcal{E}_{j}(\tau, s)=a_{0}(y, s)+\sum_{n \neq 0} a_{n}(y, s) \exp (2 \pi i n x),
$$

are the following:

$$
\begin{aligned}
a_{0}(y, s)= & y^{s} f_{0}^{(3)}(s)+y^{3-s} f_{0}^{(3)}(3-s) \\
& +8 \sqrt{\pi} \sum_{d \geq 1,(3, d)=1} d^{1 / 2} \\
& \times\left(3 \sum_{\substack{m>0 \\
(3, m)=1}} m^{-1 / 4} r_{f}(m) L^{*}(s-1,-3 m) y^{3 / 2} K_{s-3 / 2}(2 \pi d \sqrt{m} y)\right. \\
& +3^{3 / 4} \sum_{m>0} m^{-1 / 4} r_{f}(3 m) \\
& \left.\times\left(L^{*}(s-1,-9 m)+3 L^{*}(s-1,-3 m)\right) y^{3 / 2} K_{s-3 / 2}(2 \pi d \sqrt{3 m} y)\right)
\end{aligned}
$$

and

$$
\begin{aligned}
a_{n}(y, s)= & 4 \sqrt{\pi} \sum_{\substack{d \mid n \\
(3, d)=1}} d^{1 / 2}\left(3 \sum_{\substack{m>0 \\
(3, m)=1}} m^{-1 / 4} r_{f}\left(m-\frac{n^{2}}{d^{2}}\right)\right. \\
& \times L^{*}(s-1,-3 m) y^{3 / 2} K_{s-3 / 2}(2 \pi d \sqrt{m} y) \\
& +3^{3 / 4} \sum_{m>0} m^{-1 / 4} r_{f}\left(3 m-\frac{n^{2}}{d^{2}}\right) \\
& \left.\times\left(L^{*}(s-1,-9 m)+3 L^{*}(s-1,-3 m)\right) y^{3 / 2} K_{s-3 / 2}(2 \pi d \sqrt{3 m} y)\right),
\end{aligned}
$$

where

$$
r_{f}(m)=r\left(12 a^{2}+12 a c+4 c^{2}, m\right)
$$

is the number of representations of the natural number $m$ by the binary quadratic form $f(a, b)=12 a^{2}+12 a c+4 c^{2}$.

We have calculated above the Mellin transform of the Eisenstein series. Now we shall calculate the Mellin transform of the constant term of the function $\mathcal{E}_{\ell}(\tau, s)$ using Zagier's theorem (see [Za2]). Let us take for simplicity the case of the full modular group with $n(\ell)=1$. By the main theorem of [Za2] the Rankin-Selberg transform of $\mathcal{E}_{\ell}(\tau, s)(s \neq 0,3,3 / 2)$ is defined by

$$
\mathcal{R}_{\ell}(s, t)=\int_{0}^{\infty}\left(a_{0}(y, s)-\phi(y, s)\right) y^{t-2} d y
$$

where $a_{0}(y, s)$ is the constant term of the function $\mathcal{E}_{\ell}(\tau, s)$ and $\phi(y, s)=$ 
$y^{s} f_{0}^{(D)}(s)+y^{3-s} f_{0}^{(D)}(3-s)$ (the integral converges for Re $t$ sufficiently large). $\mathcal{R}_{\ell}(s, t)$ can be meromorphically continued to all complex $t$, the only possible poles being at

$$
t=0,1, s, 1-s, 3-s, 2-s \text { and } \varrho / 2
$$

( $\varrho$ is any non-trivial zero of the Riemann zeta-function) and satisfies a functional equation

$$
\mathcal{R}_{\ell}^{*}(s, t)=\mathcal{R}_{\ell}^{*}(s, 1-t), \quad \text { where } \mathcal{R}_{\ell}^{*}(s, t)=\zeta^{*}(2 t) \mathcal{R}_{\ell}(s, t) .
$$

By direct calculations we see that the integral $R\left(\mathcal{E}_{\ell}, t\right)$ is equal to

$$
\begin{aligned}
\pi^{-t} \Gamma\left(\frac{t-s}{2}+\right. & 1) \Gamma\left(\frac{t+s-1}{2}\right) \zeta^{(D)}(t) \\
& \times \sum_{\substack{u \in \widehat{R_{\ell}(0)} \\
u \neq 0}} n(2 u)^{-(t+1) / 2} \sum_{l \mid \frac{D}{q(2 u)}} l L^{*}\left(s-1,-\frac{n(2 u) D^{2}}{l^{2}}\right),
\end{aligned}
$$

where $\widehat{R_{\ell}^{(0)}}$ is the orthogonal complement to the quaternion $\ell$ in the lattice $\widehat{R^{(0)}}$ with respect to the product $\operatorname{tr}(u \bar{v})$. As a corollary we have the following

THEOREM 4.6. Let $\ell$ be a quaternion unit in the lattice $\widehat{R^{(0)}}$. The following Dirichlet series $(s \neq 0,3)$ has a meromorphic continuation to the whole complex $s$ - and t-planes:

$$
\begin{aligned}
\mathcal{R}_{\ell}^{*}(s, t)= & \pi^{-t} \Gamma\left(\frac{t-s}{2}+1\right) \Gamma\left(\frac{t+s-1}{2}\right) \zeta^{(D)}(t) \zeta^{*}(2 t) \\
& \times \sum_{m>0, D m \in \mathbb{Z}} \frac{\left.r \widehat{R_{\ell}^{(0)}}(m) \sum_{l}\right|_{\frac{D}{q(m)}} l L^{*}\left(s-1,-m D^{2} / l^{2}\right)}{m^{(t+1) / 2}},
\end{aligned}
$$

where $q(m)$ is the denominator of the rational number $m$, and

$$
r \widehat{R_{\ell}^{(0)}}(m)=\#\left\{u \in \widehat{R^{(0)}} \mid n(2 u)=m, \operatorname{tr}(u \bar{\ell})=0\right\}
$$

is the number of representations of the rational number $m$ as the norm of an element $2 u$ in the orthogonal complement $\widehat{R_{\ell}^{(0)}}$. The series is invariant with respect to the substitution

$$
t \rightarrow 1-t, \quad s \rightarrow 3-s,
$$

and could have poles with respect to $t$ only for

$$
t=0,1, s, 1-s, 3-s, s-2 .
$$


Moreover, for $s \neq 0,3,3 / 2$,

$$
\begin{aligned}
\mathcal{R}_{\ell}^{*}(s, t)= & f_{0}^{(D)}(s)\left(\frac{\zeta^{*}(2 t)}{1-s-t}+\frac{\zeta^{*}(2 t-1)}{t-s}\right) \\
& +f_{0}^{(D)}(3-s)\left(\frac{\zeta^{*}(2 t)}{s-t-2}+\frac{\zeta^{*}(2 t-1)}{t+s-3}\right) \\
& +\frac{\text { entire function of } t}{t(t-1)} .
\end{aligned}
$$

Remark 4.7. It is possible to describe the pole at $s=3 / 2$. For that we need a variant of Kronecker's summation formula for the Eisenstein series $E_{R}^{*}(z, s)$ with a logarithmic term in it. See $[\mathrm{Kr}]$ where such a formula was constructed in the case of the quaternion algebra with $D=2$. The general case is analogous.

EXAMPLE 4.8. We have the following particular formulae for the RankinSelberg transforms. In the case of the algebra with discriminant two,

$$
\begin{aligned}
\mathcal{R}_{i}^{*}(s, t)= & { }^{-t} \Gamma\left(\frac{t-s}{2}+1\right) \Gamma\left(\frac{t-s+1}{2}\right) \zeta^{(2)}(t) \zeta^{*}(2 t) \\
& \times \sum_{m>0} \frac{r\left(a^{2}+b^{2}, m\right)\left(L^{*}(s-1,-4 m)+2 L^{*}(s-1,-m)\right)}{m^{(t+1) / 2}} .
\end{aligned}
$$

For the algebra with discriminant 3 (see Example 3.4) and $\ell=i$ we have

$$
\begin{aligned}
\mathcal{R}_{j}^{*}(s, t)= & { }^{-t} \Gamma\left(\frac{t-s}{2}+1\right) \Gamma\left(\frac{t-s+1}{2}\right) \zeta^{(3)}(t) \zeta^{*}(2 t) \\
& \times\left(\sum_{\substack{m>0 \\
(m, 3)=1}} \frac{r\left(4 a^{2}+b^{2}, m\right) L^{*}(s-1,-3 m)}{(m / 3)^{(t+1) / 2}}\right. \\
& \left.+\sum_{m>0} \frac{r\left(4 a^{2}+b^{2}, 3 m\right)\left(L^{*}(s-1,-9 m)+3 L^{*}(s-1,-m)\right)}{m^{(t+1) / 2}}\right) .
\end{aligned}
$$

Analogously to Lemma 4.1, for any two orthogonal quaternions without real parts $(\operatorname{tr} \ell=\operatorname{tr} m=\operatorname{tr} \ell m=0)$ it is possible to construct the embedding of the three-dimensional hyperbolic plane $\mathcal{H}_{3}=\{(r, w) \mid r>0, w \in \mathbb{C}\}$ in the four-dimensional plane $\mathcal{H}$.

The restriction of the Eisenstein series to the subspace $\mathcal{H}_{3}$ is an invariant function with respect to a congruence subgroup of the group $\mathrm{SL}_{2}(\mathfrak{O})$, where $\mathfrak{O}$ is the ring of integers in the quadratic field $\mathbb{Q}(\ell)$. In this paper we give only one example.

EXAMPLE 4.9. Take the quaternion algebra with $D=2, \ell=i$ and $m=j$. The restriction of $E^{*}(z, s)$ is the following: 


$$
\begin{aligned}
\mathcal{E}_{i, j}((r, w), s) & \\
= & r^{s} f_{0}^{(2)}(s)+r^{3-s} f_{0}^{(2)}(3-s) \\
& +4 \sqrt{\pi} \sum_{\substack{a \in \mathbb{Z}, \alpha \in \mathbb{Z}[i] \\
(a, \alpha) \neq 0}}\left(a^{2}+\bar{\alpha} \alpha\right)^{-1 / 4} \\
& \times \sum_{\substack{t \mid(a, \alpha) \\
(t, 2)=1}}\left(t L^{*}\left(s-1,-4 \frac{a^{2}+\bar{\alpha} \alpha}{t^{2}}\right)+2 t L^{*}\left(s-1,-\frac{a^{2}+\bar{\alpha} \alpha}{t^{2}}\right)\right) \\
& \times r^{3 / 2} K_{s-3 / 2}\left(2 \pi r \sqrt{a^{2}+\bar{\alpha} \alpha}\right) \exp (\pi i \operatorname{tr}(\alpha \bar{w})) .
\end{aligned}
$$

This function is invariant with respect to the group $\mathrm{SL}_{2}(\mathbb{Z}[i])$. Its zeroth Fourier coefficient is equal to

$$
\begin{aligned}
r^{s} f_{0}^{(2)}(s)+r^{3-s} f_{0}^{(2)}(3-s) & \\
+8 \sqrt{\pi} \sum_{a>0} a^{-1 / 2} & \sum_{\substack{t \mid a \\
(t, 2)=1}} t\left(L^{*}\left(s-1,-\frac{4 a^{2}}{t^{2}}\right)\right. \\
& \left.+2 L^{*}\left(s-1,-\frac{a^{2}}{t^{2}}\right)\right) r^{3 / 2} K_{s-3 / 2}(2 \pi a r) .
\end{aligned}
$$

Acknowledgments. This research was begun while both authors were at SFB 343 at Universität Bielefeld. We wish to thank Mathematisches Institut der Georg-August-Universität and Fakultat für Mathematik der Universität Bielefeld for their hospitality. We are also indebted to Prof. J. Mennicke and Prof. U. Christian for useful conversations and interest in this paper.

\section{References}

[BS] S. Böcherer and R. Schulze-Pillot, The Dirichlet series of Koecher and Maaß and modular forms of weight 3/2, Math. Z. 209 (1992), 273-287.

[Bo] A. Borel, Some finiteness properties of adele groups over number fields, Publ. Math. IHES 16 (1963), 5-30.

[Ch] C. Chevalley, L'arithmétique dans les algèbres des matrices, Act. Sci. et Ind. 323, Hermann, Paris, 1936.

[Co] H. Cohen, Sums involving the values at negative integers of L-functions of quadratic characters, Math. Ann. 217 (1975), 271-285.

[E] M. Eichler, The basis problem for modular forms and the trace of the Hecke operators, in: Modular Functions of One Variable 1, Lecture Notes in Math. 320, Springer, Berlin, 1973, 75-151.

[EGM] J. Elstrodt, F. Grunewald, and J. Mennicke, Eisenstein series on threedimensional hyperbolic space and imaginary quadratic number fields, J. Reine Angew. Math. 360 (1985), 160-213. 
[Gr1] V. Gritsenko, Arithmetic of quaternions and Eisenstein series, Zap. Nauchn. Sem. LOMI 160 (1987), 82-90 (in Russian); English transl.: J. Soviet Math. 52 (1990), 3056-3062.

[Gr2] - Zeta-function of degree six of Hermitian modular forms of genus 2, Zap. Nauchn. Sem. LOMI 154 (1986), 46-66 (in Russian); English transl.: J. Soviet Math. 43 (1988), 2540-2553.

[Kr] A. Krieg, Eisenstein series on the four-dimensional hyperbolic space for $\operatorname{Sp}(1, \mathbb{H})$, J. Number Theory 30 (1988), 177-198.

[L] H. Leptin, Die Funktionalgleichung der Zeta-Funktion einer einfachen Algebra, Abh. Math. Sem. Hamburg 19 (1955), 198-221.

[Ma] H. Maass, Automorphe Funktionen von mehreren Veränderlichen und Dirichletsche Reihen, ibid. 16 (1949), 72-100.

[Vi] M.-F. Vigneras, Arithmétique des algèbres de quaternions, Lecture Notes in Math. 800, Springer, Berlin, 1980.

[Za1] D. B. Zagier, Modular forms whose Fourier coefficients involve zeta functions of quadratic fields, in: Modular Functions of One Variable 6, Lecture Notes in Math. 627, Springer, Berlin, 1977, 105-169.

[Za2] - The Rankin-Selberg method for automorphic functions which are not of rapid decay, J. Fac. Sci. Univ. Tokyo 28 (1981), 415-437.

ST. PETERSBURG DEPARTMENT

STEKLOV MATHEMATICAL INSTITUTE

FONTANKA 27

191011 ST. PETERSBURG, RUSSIA
MATHEMATISCHES INSTITUT DER UNIVERSITÄT ZU KÖLN WEYERTAL 86-90 D-50931 KÖLN, GERMANY 